\title{
Transient sub-nanosecond soft X-ray NEXAFS spectroscopy on or- ganic thin films
} \author{
valou $^{1,2,4}$ \\ 1) Berlin Laboratory for innovative X-ray technologies (BLiX), D-10623 Berlin, Germany \\ 2) TU Berlin, Analytical X-Ray Physics, D-10623 Berlin, Germany \\ 3) Max-Born-Institut für Nichtlineare Optik und Kurzzeitspektroskopie, D-12489 Berlin, Germany \\ 4) Current affiliation: Helmholtz Zentrum Berlin, D-12489, Germany \\ 5) Departamento de Química Física, Universidad de Sevilla, Seville ES-41012, Spain \\ *) Corresponding author, Adrian.Jonas@campus.tu-berlin.de
}

Adrian Jonas ${ }^{1,2, *}$, Katharina Dammer ${ }^{1,2}$, Holger Stiel ${ }^{1,3}$, Birgit Kanngießer ${ }^{1,2}$, Rocío Sánchez-de-Armas ${ }^{5}$ and Ioanna Mantou-

\begin{abstract}
We demonstrate visible pump soft X-ray probe NEXAFS spectroscopy measurements at the carbon K edge on thin molecular films in the laboratory. This opens new opportunities through the use of laboratory equipment for chemical speciation. We investigate the metal free porphyrin derivative tetra(tert-butyl)porphyrazine (TAP) as an ideal model system to elucidate electronic properties of tetrapyrroles like chlorophyll or heme. In contrast to measurements in gas or liquid state, the investigation of thin films is of high interest in the field of optoelectronic and photovoltaic devices though challenging due to the low damage thresholds of the samples upon excitation. With a careful pre-characterization using optical techniques, successful measurements were performed using a NEXAFS spectrometer based on a laser-produced plasma source and reflection zone plates with a resolving power of 1000 and a time resolution of $0.5 \mathrm{~ns}$. In combination with DFT calculations first insights into a long-lived excitonic state are gained and discussed.
\end{abstract}

With transient near edge X-ray absorption fine structure spectroscopy (tr-NEXAFS) element specific information of the chemical nature and electronic structure of a sample is gained. ${ }^{1-3}$ Using UV/VIS pump and soft X-ray probe pulse energies, excited state dynamics of organic molecules at the K-edges of their main constituents $(\mathrm{C}, \mathrm{N}, \mathrm{O})$ can be investigated. In tr-NEXAFS a valence excitation of a molecule is induced using visible or ultraviolet light. Whilst excited the soft $\mathrm{X}$-ray probe pulse promotes an inner shell electron into an empty valence orbital. ${ }^{4,5}$ Due to the excitation optically dark states or previously fully occupied orbitals can be investigated. ${ }^{4,6,7}$ By varying the time delay between pump and probe pulse the dynamics of the chemical configuration of the molecule is accessible. ${ }^{4,5} 8$ Depending on the light induced photophysical processes different time domains are of interest ranging from femtoseconds to milliseconds. These domains can nowadays be reached with both laboratory based and large scale facility X-ray sources. ${ }^{2,3}$ Commonly, tr-NEXAFS experiments are performed at synchrotron radiation facilities where $\mathrm{X}$-ray pulse duration and therefore time resolution of typically 20 ps to $100 \mathrm{ps}$ is available. ${ }^{2}$ By adjusting the monochromator, the X-ray energy hitting the sample is scanned in small steps. The absorption is determined by means of secondary deexcitation effects i.e. the total electron or fluorescence yield of the sample. The external pump laser must be timed to the X-ray pulse. If a high harmonic generation or laser-produced plasma source is used, the same laser is used for the pump and probe pulse, making the experiment jitter-free. The significantly smaller photon numbers of these laboratory sources make measurements in transmission mode necessary. Although this is the more direct way to obtain the absorption, it makes sample preparation more challenging. The polychromatic radiation transmits through the sample and needs to be dispersed by a dispersive element. This makes single shot experiments possible and facilitates the detection of radiation damage. ${ }^{9}$

Until now soft X-ray tr-NEXAFS investigations on molecules were mainly concentrated on gas phase experiments ${ }^{4,5,8}$ or on liquid jets ${ }^{10,11}$ or cells. ${ }^{12}$ However, in many cases (e.g. in functionalized materials for optoelectronic and photovoltaic devices) organic molecules are directly bound to a substrate or organized in thin layers. Tr-NEXAFS investigations on thin, solid layers of organic molecules are very challenging due to degradation processes after heat dissipation caused by the pump pulse. This process can be minimized if the average power of the pump pulse is reduced. However, the detectable absorption change $\Delta \mathrm{A}$ in tr-NEXAFS experiment directly depends on the intensity of the pump pulse. Therefore the interplay between heat transfer in the sample by the pump pulse and detection sensitivity for $\Delta \mathrm{A}$ must be optimized. 
In this contribution we will demonstrate the feasibility of subnanosecond tr-NEXAFS spectroscopy on a thin film of tetra(tert-butyl)porphyrazine (TAP) as a typical molecule belonging to the class of tetrapyrroles. Tetrapyrroles exhibit a great application potential in optoelectronics and photovoltaics as well as pigments in natural or artificial photosynthesis. Porphyrazines are a very versatile group of molecules because their electronic structure can be altered through modifications of the ligand or central atom. ${ }^{13,14}$ The wide range of biological and technological applications demands a complete description of the electronic properties of this class of molecules. For example the extension of the $\pi$-electron system can be easily changed with a systematic increase of benzo units through linear benzo annelation. ${ }^{14}$ The tert-butyl substitution induces only a very small change in the electronic structure at the valence region as the light absorption takes place in the delocalized $\pi$-electron system due to the conjugated carbon bonds situated in the central $\mathrm{C}_{8} \mathrm{~N}_{8}$ structure. TAP films show two distinct luminescence peaks with maxima at $695 \mathrm{~nm}$ and 628 $\mathrm{nm}$ after excitation of the first excited state indicating two different luminescent decay processes which is unusual for these kinds of systems. ${ }^{15}$ This second decay channel might originate from long-lived excitonic trap states because of its small quantum efficiency and absence in solution. ${ }^{15}$ As this state could be exploited in light-harvesting processes due to its long life-time it is an interesting sample for proof-of-principle tr-NEXAFS measurements.

Our newly developed soft X-ray tr-NEXAFS spectrometer is based on a laser-produced plasma source and operates with photon energies for the probe pulse up to $1303 \mathrm{eV}$ (Mg Kedge) with a resolving power of over 1000 and a time resolution of 0.5 ns. ${ }^{9,16,17}$ The optimal parameters for the pump light were determined by means of non-linear absorption (NLA) measurements. Transient NEXAFS spectra at the C K-edge after $515 \mathrm{~nm}$ excitation at different delay times will be shown and interpreted. The ground state NEXAFS is compared to Density Functional Theory calculations (DFT) in order to assign the characteristic NEXAFS features.

\section{EXPERIMENTAL SECTION}

The measurements were performed using a laboratory laserproduced plasma source and a reflection zone plate pair spectrometer, described in. ${ }^{16}$ A scheme of the setup is depicted in Fig. 1.

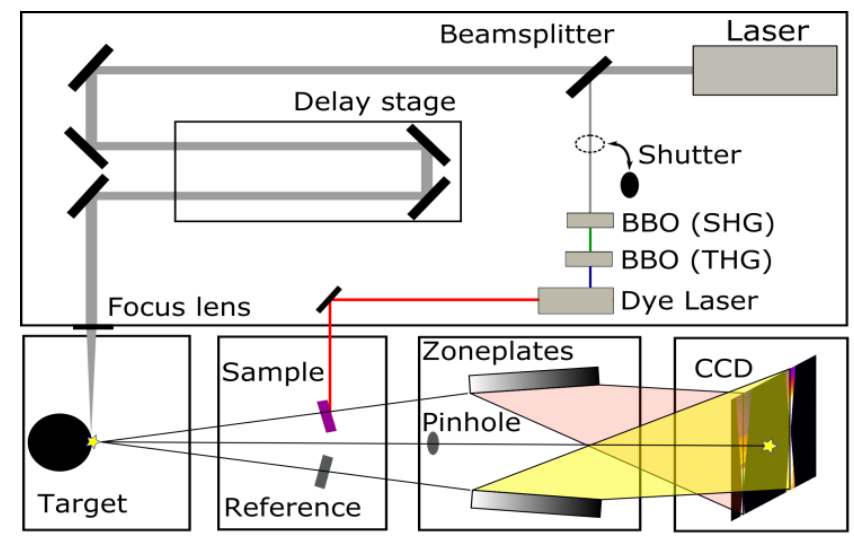

Figure 1. Schematic view of the setup. The same laser is used for the pump and the probe beam. The sample and reference spectrum is collected simultaneously with each shot of the laser.

A $150 \mathrm{~mJ}, 1030 \mathrm{~nm}, 0.5 \mathrm{~ns}$ pulse is focused onto a rotating metal cylinder with a repetition rate of $100 \mathrm{~Hz}$ and forms a hot dense plasma with a size of $50 \mu \mathrm{m}$ full width at half maximum. The plasma emits polychromatic and isotropic radiation in the range of $80 \mathrm{eV}-1600 \mathrm{eV}$. The sample is measured in transmission mode and the reference spectrum is collected simultaneously using a pair of reflection zone plate optics. In front of each zone plate a $200 \mathrm{~nm}$ thick titanium foil blocks residual laser light, the visible part of the emission spectrum and in second order diffracted light. Additionally a $100 \mathrm{~nm}$ thick aluminum foil is situated right in front of the sample to protect it from debris of the source. The dispersed light is collected using a CCD detector (Greateyes GmbH, GE 2048 $2048 \mathrm{BI})$. The source has an online stability correction system and is capable of measuring transient changes $\Delta \mathrm{A}$ in the order of $10^{-4}$. The resolving power of the spectrometer at the carbon $\mathrm{K}$-edge is about 1000 . The whole spectrometer is operated under high vacuum conditions $\left(10^{-7} \mathrm{mbar}\right)$. For the optical excitation of the sample a small a part of the plasma producing laser light is separated using a variable beamsplitter. The 1030 $\mathrm{nm}$ laser light is frequency-doubled to $515 \mathrm{~nm}$ using a BBO crystal to excite the sample resonantly in the first excited state (Qx-band). Optionally a second BBO crystal can be inserted to generate the third harmonic $(343 \mathrm{~nm})$. A dye laser can be used with an accessible wavelength range between $400 \mathrm{~nm}$ and $1000 \mathrm{~nm}$. The pump-probe delay is varied continuously by spatial separation of two probe beam mirror pairs to a maximum of $43 \mathrm{~ns}$.

The non-linear absorption (NLA) measurement was performed using the pump laser at $515 \mathrm{~nm}$ and a J-10-MB-LE laser energy sensor from Coherent. The photon flux density was adjusted by changing the output pulse energy of the laser. The spot size on the sample $(1.4 \pm 0.1 \mathrm{~mm})$ was determined by a knife edge scan.

The tr-NEXAFS spectra were taken at the C K-edge. To verify the validity of the transient changes a negative time delay of 3 ns between pump and probe pulse was chosen for the first and last measurement. While these spectra act as reference for the ground state, also long-term heating processes and scattered pump light can be evaluated. Six different time delays were probed starting at full overlap at $0 \mathrm{~ns}$ up to $43 \mathrm{~ns}$. At each time delay a total of 20.000 single shot NEXAFS images were taken with $0.4 \mathrm{~Hz}$ due to the limited readout speed of the CCD. The measurement time for each time delay was between 13-26 hours leading to a total data collection time of 4 days and 21 hours. Possible long-term instabilities of the laser or detector are suppressed by collecting the ground state and excited state NEXAFS images in an alternating mode. The absorption is obtained by taking the natural logarithm of the reference spectra divided by the sample spectra and the difference spectrum is obtained by subtracting the excited state spectrum from the ground state spectrum. A more detailed description is given in. ${ }^{16}$

TAP powder was purchased from Sigma Aldrich and used without further purification. $35 \mathrm{mg}$ of TAP were placed in a crucible and heated using an effusion cell to $210{ }^{\circ} \mathrm{C}$ inside a 
vacuum chamber at $10^{-6}$ mbar and evaporated consecutively onto three standard $10 \mathrm{~mm} \times 10 \mathrm{~mm}$ silicon frames with a 2 mm x $2 \mathrm{~mm}$ x $150 \mathrm{~nm} \mathrm{Si}_{3} \mathrm{~N}_{4}$ window (Norcada Inc.). About $99.99 \%$ of material is wasted in the process. Multiple samples were needed because of occasional damage that is caused by debris particles ejected from the plasma source. A total of ten samples were produced and measured during the campaign. Using tabulated data for X-ray mass absorption coefficients and the measured $\mathrm{C} \mathrm{K}$ absorption edge jump the sample thicknesses were estimated to be $200 \mathrm{~nm}$ for the first two samples and $100 \mathrm{~nm}$ for the other eight samples. UV/VIS spectra were taken to confirm that the molecules are not impaired during the evaporation process. TAP films are known to lack any surface effects. ${ }^{15}$

\section{RESULTS AND DISCUSSION}

In order to find the best laser parameters for the excitation of the sample UV/VIS and NLA measurements were performed. Fig 2. (left) shows the visible absorbance of one of the samples. The HOMO-LUMO transition with its two Q-bands can be seen. The photon energy used for the NLA and tr-NEXAFS measurement is shown as a vertical line. Fig 2. (right) shows an NLA measurement on the same sample. In an NLA measurement the transmission of a sample is measured in dependence on photon flux density. At high intensities the linearity of the Lambert-Beer law between incident intensity and absorbed intensity breaks down due to changes in the occupation of the participating states. This means, in the nonlinear regime, a significant portion of the molecules is in the excited state and consequently changes in the tr-NEXAFS can be expected. At photon flux densities below $2.5 * 10^{24}$ photons $/\left(\mathrm{cm}^{2 *} \mathrm{~s}\right)$, the transmission shows no dependence on photon flux density and matches the transmission obtained by the low signal UV/VIS measurement. The transmission then increases from $72.5 \%$ to $75.5 \%$ before the samples get thermally damaged at around $1.1 * 10^{25}$ photons $/\left(\mathrm{cm}^{2 *} \mathrm{~s}\right)$. The indicated error bars result from fluctuations of the laser pulse energy and uncertainties of the measuring instruments. NLA measurements were also performed using a resonant excitation of the Qy-band at $620 \mathrm{~nm}$. The dye laser (Rhodamine B) was deployed and pumped with the $515 \mathrm{~nm}$ light. The relative change in transmission at high photon flux densities were the same so that $515 \mathrm{~nm}$ was chosen for the tr-NEXAFS pump energy because its generation does not require the additional dye laser. For all tr-NEXAFS measurements a photon flux density of $5 * 10^{24}$ photons $/\left(\mathrm{cm}^{2 *} \mathrm{~s}\right)$ $\left(=1 \mathrm{~mJ} / \mathrm{cm}^{2}\right)$ was chosen.
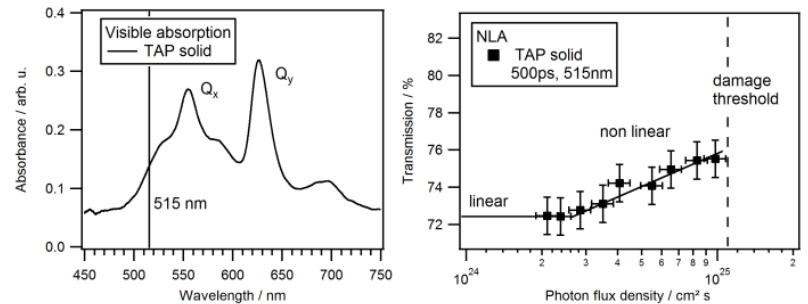

Figure 2. Left: Visible absorption spectrum of the first excited state of TAP. The vertical line highlights the excitation energy of the NLA and tr-NEXAFS measurement. Right: NLA measurement of a TAP film. The straight lines emphasize the linear and the non-linear part of the curve. The dashed line shows the photon flux density at which sample degradation takes place.

The C K-edge NEXAFS spectrum was calculated for each carbon atom individually using the gradient-corrected DFT Program StoBe ${ }^{18}$ with the exchange functional by Becke ${ }^{19}$ and the correlation functional by Perdew. ${ }^{20}$ The TAP geometry was firstly optimized using the hybrid exchange-correlation functional B3LYP as implemented in the Gaussian 09 package with $6-31 \mathrm{G}^{* *}$ basis set. ${ }^{21}$ The excited $1 \mathrm{~s}$ state was represented as a half core-hole according to the transition potential method. ${ }^{22}$ The energy positions of the single-atom NEXAFS curves were corrected according to the corresponding computed IP ( $\triangle \mathrm{KS}-\mathrm{SCF}$ correction). The spectral intensities were generated from the computed dipole transition probabilities and the results were then convoluted by Gaussian curves. For each $\mathrm{C}$ separately, a Gaussian broadening with a fixed full width at half-maximum (FWHM) was used in the low energy part of the spectrum $(0.5 \mathrm{eV})$, while, starting from the IP, the FWHM was linearly increased from the initial value of $0.5 \mathrm{eV}$ up to 5 $\mathrm{eV}$ in order to better reproduce the experimental continuum region. The total calculated NEXAFS spectrum for TAP molecule was obtained by summing up all the single-atom $1 \mathrm{~s}$ theoretical spectra. Moreover, the calculations yield spectra for different classes of carbon atoms within the structure, see Fig. 3.

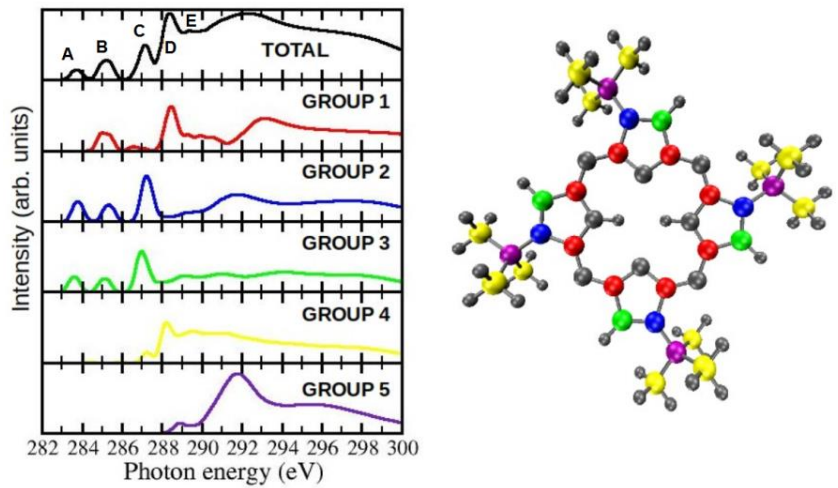

Figure 3. Left: Calculated C K-edge NEXAFS for the whole TAP molecule and isolated contributions from the different groups of atoms. Right: TAP structure with carbon atoms represented in different colors depending on the assigned group. All species different from carbon are represented in grey color.

Table. 1. Description of the different carbon groups.

\begin{tabular}{|l|l|}
\hline Group & Description \\
\hline 1 red & C bound to $\mathrm{N}$ \\
\hline 2 blue & C bound to three C within ring (sp2) \\
\hline 3 green & CH bound to two C within ring (sp2) \\
\hline 4 yellow & CH3 from butyl group (sp2) \\
\hline 5 purple & C bound to three C from butyl groups (sp3) \\
\hline
\end{tabular}

From Fig. 3 it can be seen that peak A consist of contributions from groups 2 and 3, i.e. carbon atoms bound to two or three carbon atoms within the ring. All these carbon atoms have a 
common feature, they have one double bond and sp2 hybridization. Peak B has also contributions from the same carbons as peak $\mathrm{A}$ but it also has an important contribution from carbon atoms that are bound to nitrogen atoms (group 1). Peak $\mathrm{C}$ has contributions from the same groups of carbons than peak A (mainly 2 and 3). Peak D has contributions from groups 1 and 4 i.e. carbon atoms bound to nitrogen and the carbons of the tert-butyl substitution with $\mathrm{sp} 3$ hybridization. Finally, the weak peak E origins from Group 1.
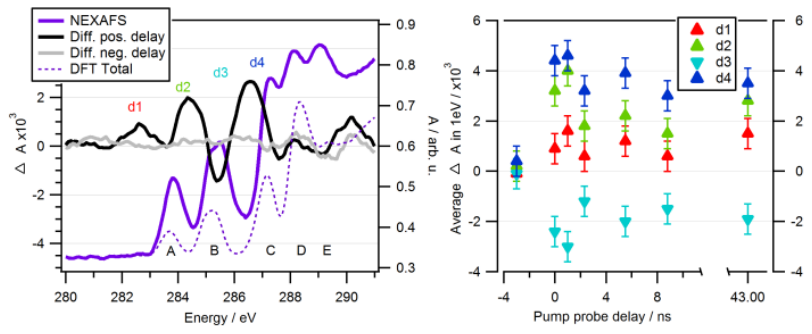

Figure 4. Left: The ground state NEXAFS spectrum is shown in purple. Two difference spectra are shown in grey $(-3 \mathrm{~ns}$ delay) and black (Average of all pos. delays). Right: The differences of the for regions d1 to $\mathrm{d} 4$ at different time delays.

The result of the tr-NEXAFS measurement is shown in Fig. 4. The measurement was calibrated using isolated copper plasma emission lines leading to an uncertainty of the energy calibration of $0.2 \mathrm{eV} .^{9}$ Absolut peak positions are listed in table 2 . Direct comparison of the ground state NEXAFS with the calculated spectra shows that the absolute peak positions are reproduced exceptionally well in the calculation. While the relative intensities of peaks A to D are in good agreement, peak $\mathrm{E}$ is stronger in the measurement.

Table. 2. Comparison of calculated and measured peak positions.

\begin{tabular}{|l|l|l|}
\hline Peak & Calculated energy / eV & Measured energy / eV \\
\hline A & 283.7 & $283.9 \pm 0.2$ \\
\hline B & 285.2 & $285.4 \pm 0.2$ \\
\hline C & 287.2 & $287.4 \pm 0.2$ \\
\hline D & 288.4 & $288.2 \pm 0.2$ \\
\hline E & 288.9 & $289.0 \pm 0.2$ \\
\hline
\end{tabular}

The absorption changes in dependence on pump-probe delay are depicted at Fig. 4, right. These changes remain nearly constant, even at very long delay times suggesting a steady state behavior of the electronic transitions in the molecule after excitation with the $0.5 \mathrm{~ns}$ pulse (see below). Therefore we averaged the difference spectra of all positive time delays for better statistics (black curve in Fig. 4, left). In order to validate the data at longer delay times the spectrum with negative pump-probe delay is shown in grey. Both difference spectra are smoothed into $1 \mathrm{eV}$-bins using a $(15 \mathrm{x})$ box filter. The difference spectrum with negative delay shows a standard deviation of $2 * 10^{-4}$ in the pre edge region $(250 \mathrm{eV}-283 \mathrm{eV})$ and $4 * 10^{-4}$ starting at feature $\mathrm{A}(283 \mathrm{eV}-300 \mathrm{eV})$, which is a measure for the uncertainty $\Delta \mathrm{A} . \Delta \mathrm{A}$ is nevertheless estimated to be $\pm 5^{*} 10^{-4}$ because of errors originating from sample thickness and pump pulse stability. The biggest light induced changes can be seen between peak $A$ and $B(d 2)$ at peak B (d3) and between peak B and C (d4). While the absorption at $\mathrm{d} 1, \mathrm{~d} 2$ and $\mathrm{d} 4$ is higher for the excited sample it is slightly lower at d3. Difference d1 possibly arises from an X-ray optical double resonance i.e. a carbon $1 \mathrm{~s}$ electron transitioning into the partially depleted HOMO of the excited molecules. ${ }^{4,23}$ Difference $\mathrm{d} 3$ is a reduction of peak $\mathrm{B}$, which originates mainly from carbon atoms that are bound to nitrogen atoms. It can be assumed that the reduction of density of unoccupied states is transferred from $\mathrm{d} 3$ to $\mathrm{d} 1$ in the excited state. Differences $\mathrm{d} 2$ and $\mathrm{d} 4$ are in between peak A, B and C. While $\mathrm{d} 2$ might also arise partly from a shift in density of unoccupied states of group B atoms, the area of $\mathrm{d} 2$ is much larger than the area of p3 and therefore, like $\mathrm{d} 4$, must have other contributions that cannot be isolated easily. The absorption of the butyl groups (group 4 and 5) seem unchanged in the excited state which is in accordance with the UV/VIS measurement. ${ }^{15}$

As previously stated, the time evolution of the differences is depicted in Fig. 4 (right). For each delay, the depicted values are the extremum of each region $\mathrm{d} 1$ to $\mathrm{d} 4$, which are equal to the average absolute difference in a $1 \mathrm{eV}$ bin around the regions center. Within this uncertainty, differences do not seem to decline significantly in the investigated delay range, indicating a long lived state.

\section{CONCLUSION}

The carbon K-edge NEXAFS of a thin organic molecule film was measured in the excited state with sub-nanosecond time resolution. This proof-of-principle measurement was performed using a laboratory setup based on a laser-produced plasma source and a reflection zone plate spectrometer. The excitation was optimized with preceding non-linear absorption measurements rendering experiments below the damage threshold of the sample possible. In order to assign the NEXAFS features to the respective carbon groups, DFT calculations were conducted. A slow decay channel that is only apparent in solid TAP films could be investigated. The density of unoccupied states for carbon atoms that are bound to nitrogen atoms are slightly reduced while the contributions from the butyl groups remain unchanged. The life time of this long living transient state can only be estimated to be longer than 43 ns. To further elucidate the transient changes and deepen the understanding of the electronic structure measurements with longer delays are planned as well as visible pump soft $\mathrm{X}$ ray probe measurements at the $\mathrm{N} \mathrm{K}$-edge. Measurement times could be reduced by a factor of 250 if the full $100 \mathrm{~Hz}$ of the system could be exploited e.g. when 16-bit back illuminated scientific CMOS cameras with higher readout speed, adequate pixel and sensor size become readily available.

The combination of careful pre-characterization using optical techniques such as UV/VIS and NLA spectroscopy and the possibility to use lab-based spectrometers enables low-dose experiments which are a prerequisite when dealing with solid state samples. Additionally, the use of equipment which is independent on large scale facilities and in principle available for any analytics laboratory, enables comprehensive chemical characterization for material development and monitoring. 
Thus, by demonstrating the possibility of the investigation of the dynamic electronic properties of organic thin films, this proof-of-principle experiment opens up the tr-NEXAFS technique to a whole new class of samples and applications.

\section{AUTHOR INFORMATION}

\section{Corresponding Author}

*E-mail: Adrian.Jonas@ campus.tu-berlin.de

\section{Author Contributions}

The manuscript was written through contributions of all authors. All authors have given approval to the final version of the manuscript.

\section{Notes}

The authors declare no conflicts of interest. The data that support the findings of this study are available from the corresponding author upon reasonable request.

\section{ACKNOWLEDGMENT}

This work was funded by the Deutsche Forschungsgemeinschaft (313838950) and LASERLAB Europe (654148). R. Sánchez-deArmas thanks VPPI-US and project PGC2018-101689-B-I00 (MCI/AEI/FEDER,UE) for the financial support. The access to the computational facilities of the "Centro de Servicios de Informática y Redes de Comunicaciones" (CSIRC, Universidad de Granada, Spain) are also acknowledged. Figure 3. was created using VMD. ${ }^{24}$

\section{REFERENCES}

(1) Fushitani, M. Applications of pump-probe spectroscopy. Annu. Rep. Prog. Chem., Sect. C: Phys. Chem. 2008, 104, 272-297.

(2) Bressler, C.; Chergui, M. Ultrafast X-ray Absorption Spectroscopy. Chem. Rev. 2004, 104, 4, 1781-1812.

(3) Chen, L. X. Probing transient molecular structures in photochemical processes using laser-initiated time-resolved X-ray absorption spectroscopy. Annu. Rev. Phys. Chem. 2005, 56:221-254.

(4) Wolf, T. J. A.; Myhre, R. H.; Cryan, J. P.; Coriani, S.; Squibb, R. J.; Battistoni, A.; Berrah, N.; Bostedt, C.; Bucksbaum, P.; Coslovich, G.; Feilfe, R.; Gaffney, K. J.; Grilj, J.; Martinez, T. J.; Miyabe, S.; Moeller, S. P.; Mucke, M.; Natan, A.; Obaid, R.; Osipov, T.; Plekan, O.; Wang, S.; Koch, H.; Gühr, M. Probing ultrafast $\pi \pi^{*} / \mathrm{n} \pi^{*}$ internal conversion in organic chromophores via K-edge resonant absorption. Nat. Commun. 2017, 8, 29.

(5) Attar, A. R.; Bhattacherjee, A.; Pemmaraju, C. D.; Schnorr, K.; Closser, K. D.; Prendergast, D.; Leone, S. R. Femtosecond x-ray spectroscopy of an electrocyclic ring-opening reaction. Science. 2017, 356(6333): 5459 .

(6) Stiel, H.; Leupold, D.; Beck, M.; Will, I.; Lokstein, H.; Sandner, W. Towards time resolved, coupled structure-function information on carotenoid excited state processes: X-ray and optical short pulse double resonance spectroscopy. J. Biomed. Biophys. Methods. 2001, 48:239-246.

(7) Hua, W.; Mukamel, S.; Luo, Y. Transient X-ray Absorption Spectral Fingerprints of the S1 Dark State in Uracil. J. of Phys. Chem. Lett. 2019, $10,22,7172-7178$.

(8) Pertot, Y.; Schmidt, C.; Matthews, M.; Chauvet, A.; Huppert, M.; Svoboda, V.; Conta, A. von,; Tehlar, A.; Baykusheva, D.; Wolf, J.-P.; Wörner, H. J. Time-resolved x-ray absorption spectroscopy with a water window high-harmonic source. Science 2017, 355(6322), 264-267.
(9) Mantouvalou, I.; Witte, K.; Wartyanov, W.; Jonas, A.; Grötzsch, D.; Streeck, C.; Löchel, H.; Rudolph, I.; Erko, A.; Stiel, H.; Kanngießer, B. Single shot near edge $\mathrm{x}$-ray absorption fine structure spectroscopy in the laboratory. Appl. Phys. Lett. 2016, 108(20), 201106.

(10) Fondell, M.; Eckert, S.; Jay, R. M.; Weniger, C.; Quevedo, W.; Niskanen, J.; Kennedy, B.; Sorgenfrei, F.; Schick, D.; Giangrisostomi, E.; Ovsyannikov, R.; Adamczyk, K.; Huse, N.; Wernet, P.; Mitzner, R. Föhlisch, A. Time-resolved soft X-ray absorption spectroscopy in transmission mode on liquids at $\mathrm{MHz}$ repetition rates. Structural Dynamics. 2017, 4(5): 054902

(11) Kleine, C.; Ekimova, M.; Goldsztejn, G.; Raabe, S.; Strüber, C.; Ludwig, J.; Yarlagadda, S.; Eisebitt, S.; Vrakking, M. J. J.; Elsaesser, T.; Nibbering, E. T. J.; Rouzée, A. Soft X-ray Absorption Spectroscopy of Aqueous Solutions Using a Table-Top Femtosecond Soft X-ray Source. $J$. of Phys. Chem. Lett. 2019, 10, 1, 52-58.

(12) Van Kuiken, B. E.; Cho, H.; Hong, K.; Khalil, M.; Schoenlein, R. W.; Kim, T. K.; Huse, N. Time-Resolved X-ray Spectroscopy in the Water Window: Elucidating Transient Valence Charge Distributions in an Aqueous Fe(II) Complex. J. Phys. Chem. Lett. 2016, 7, 3, 465-470.

(13) Nardi, M. V.; Detto, F.; Aversa, L.; Verucchi, R.; Salviati, G.; Iannotta, S.; Casarin, M. Electronic properties of CuPc and H2Pc: an experimental and theoretical study. Phys. Chem. Chem. Phys. 2013, 15, 1286412881.

(14) Pop, D.; Winter, B.; Freyer, W.; Hertel, I. V.; Widdra, W. Electronic Structure of Metal-Free Porphyrazines in Thin Films. J. Phys. Chem. B. 2003, 107, 42, 11643-11647.

(15) Freyer, W.; Neacsu, C. C.; Raschke, M. B. Absorption, luminescence, and Raman spectroscopic properties of thin films of benzo-annelated metal-free porphyrazines. Journal of Luminescence. 2008, 128, 661-672.

(16) Jonas, A.; Stiel, H.; Glöggler, L.; Dahm, D.; Dammer, K.; Kanngießer, B.; Mantouvalou, I. Towards Poisson limited optical pump soft X-ray probe NEXAFS spectroscopy using a laser-produced plasma source. Optics Express. 2019, 27(25), 36524-36537.

(17) Mantouvalou, I.; Witte, K.; Grötzsch, D.; Neitzel, M.; Günther, S.; Baumann, J.; Jung, R.; Stiel, H.; Kanngießer, B.; Sandner, W. High average power, highly brilliant laser-produced plasma source for soft Xray spectroscopy. Rev. Sci. Instrum. 2015, 86(3), 035116.

(18) Hermann, K.; Petterson, L. StoBe-deMon, version 3.0, 2007.

(19) Becke, A. D. Density-functional exchange-energy approximation with correct asymptotic behavior. Phys. Rev. A 1988, 38, 3098.

(20) Perdew, J. P. Density-functional approximation for the correlation energy of the inhomogeneous electron gas. Erratum Phys. Rev. B, 1986, $34,7406$.

(21) Frisch, M. J.; Trucks, G. W.; Schlegel, H. B.; Scuseria, G. E.; Robb, M. A.; Cheeseman, J. R.; Scalmani, G.; Barone, V.; Petersson, G. A. Nakatsuji, H. et al. Gaussian 09, Revision C.01, Gaussion Inc.: Wallingford CT, 2016.

(22) Triguero, L.; Pettersson, L. G. M.; Ågren, H. Calculations of nearedge $\mathrm{x}$-ray-absorption spectra of gas-phase and chemisorbed molecules by means of density-functional and transition-potential theory. Phys. Rev. B. 1998, 58, 8097. 
(23) Vogt, U.; Wilhein, T.; Legall H.; Stiel, H. High-Resolution X-Ray Absorption Spectroscopy Using a Laser Plasma Radiation Source. IPAP Conf. Series 7, 2006, 334.

(24) Humphrey, W.; Dalke A.; Schulten, K.; Visual Molecular Dynamics. J. Molec. Graphics, 1996, 14.1, 33-38. 


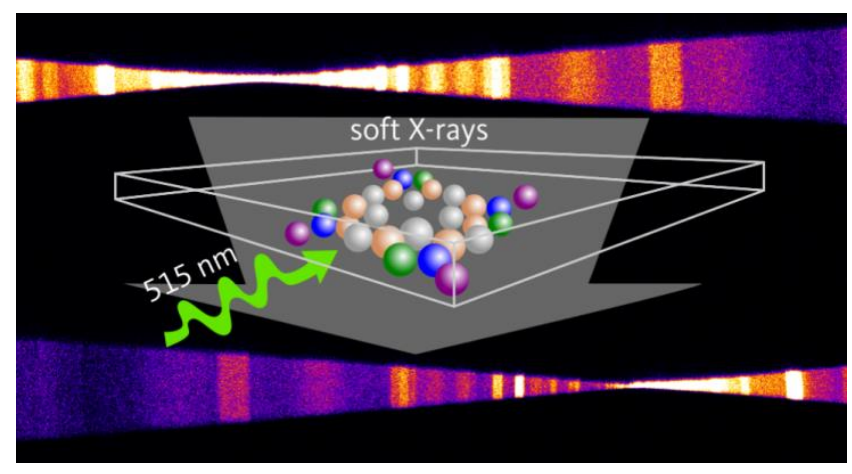

"For Table of Contents Only" 